\title{
Prognostic significance of programmed ventricular stimulation in survivors of acute myocardial infarction
}

\author{
ANIL K BHANDARI, ROBERT HONG, ADAM KOTLEWSKI, \\ NANCY MCINTOSH, PHILLIP AU, ANTHONY SANKOORIKAL, \\ SHAHBUDIN H RAHIMTOOLA
}

From the Section of Cardiology, Department of Medicine, LAC-USC Medical Center, University of Southern California School of Medicine, Los Angeles, California, USA

SUMMARY The prognostic significance of the response to programmed ventricular stimulation was studied in 75 stable survivors of acute myocardial infarction. Programmed ventricular stimulation induced sustained ventricular arrhythmias in $33(44 \%)$ patients and did not induce these arrhythmias in $42(56 \%)$ patients. During a mean follow up of 18 months, four patients died suddenly and three developed spontaneous sustained ventricular tachycardia. The occurrence of arrhythmic events was not significantly different in patients with inducible sustained arrhythmias and those without, but such events were predicted by the presence of mild congestive heart failure. Although the inducibility of sustained ventricular tachycardia (but not ventricular fibrillation) seemed to identify a high risk subset with an arrhythmic event rate of $21 \%$ compared with $5.5 \%$ in others, it had a low sensitivity $(57 \%)$ and a low positive predictive accuracy $(21 \%)$ for arrhythmic events.

Programmed ventricular stimulation is not helpful in identifying a subset of patients at high risk after an uncomplicated acute myocardial infarction.

In survivors of acute myocardial infarction mortality in the first year after discharge from hospital varies from 7 to $11 \%{ }^{1-3}$ Sudden cardiac death accounts for about half of the deaths and is probably caused by ventricular tachycardia or fibrillation. In recent years several investigators have examined the value of programmed ventricular stimulation in identifying a subset at an increased risk of sudden death, ${ }^{4-11}$ but the results have been conflicting. Moreover, none of the reported studies used a standard stimulation protocol of three extrastimuli or compared the prognostic significance of inducible ventricular arrhythmias with that of other prognostic variables.

We assessed the prognostic value of the induced response to programmed ventricular stimulation in a prospective study of clinically stable survivors of acute myocardial infarction. We also used a stepwise

Requests for reprints to Dr Anil K Bhandari, USC School of Medicine, Section of Cardiology, 2025 Zonal Avenue, Los Angeles, CA 90033, USA.

Accepted for publication 20 December 1988
Cox regression analysis to assess the value of programmed ventricular stimulation against other known clinical, electrocardiographic, and angiographic prognostic variables.

\section{Patients and methods}

Patients aged $\leqslant 70$ years admitted to the intensive cardiac care unit of our hospital with a diagnosis of an $\frac{D}{0}$ acute myocardial infarction were screened for the study. Myocardial infarction was diagnosed accord- N ing to clinical, electrocardiographic, and enzymatic criteria. Patients were eligible if they were clinically stable and did not have Killip class III or IV $\omega$ congestive heart failure, moderate to severe angina pectoris, or spontaneous sustained ventricular tachycardia in the $\mathbf{4 8}$ hours after myocardial infarction. Patients were excluded if they had end stage ? disease of another organ system or if they refused to participate in the study.

From September 1983 to June 1985, 164 patients with a confirmed diagnosis of acute myocardial infarction were eligible for the study. There were 17 
deaths in hospital. Seventy two patients were excluded by predetermined criteria (table 1). We studied the remaining $75(51 \%)$ consenting patients (mean age 53 (9) years (range 35 to 67 years)), including the 45 patients whose initial preliminary findings have been previously reported. ${ }^{10}$

The infarct was anterior in 36 patients $(48 \%)$ and inferior in 39 patients (52\%). Fourteen patients had mild congestive heart failure and 16 had mild angina pectoris. The mean peak creatine kinase activity was 1928 (1607) IU/1 (range 250-7250 IU/1) with an MB isoenzyme fraction of $16(9) \%(7-35 \%)$. The medications at the time of the study included digoxin in nine patients, diuretics in 14 patients, nitrates in 56 patients, and calcium channel antagonists in 12 patients.

\section{METHODS}

After giving their written informed consent patients had 24 hour ambulatory electrocardiographic monitoring, programmed ventricular stimulation, and cardiac catheterisation with coronary angiography. These tests were performed within 7-18 (11 (3)) days after the infarct. Programmed ventricular stimulation and cardiac catheterisation were performed together on the same day after an average of 14 (5) days (9-21 days) after the infarct.

Programmed ventricular stimulation was performed with patients in the postabsorptive and unsedated state. Surface electrocardiographic leads (V1, I, and aVF) and intracardiac electrograms were displayed and recorded on an oscilloscopic photographic recorder (Electronics for Medicine, VR-16) at a paper speed of $25 \mathrm{~mm} / \mathrm{s}$. Stimuli of $2 \mathrm{~ms}$ at twice the diastolic threshold (minimum pacing current, $1.5 \mathrm{~mA}$ ) were provided by a digital programmable stimulator (Bloom and Associates). The stimulation protocol was uniform in each patient and has been described in detail previously. ${ }^{10}$ Briefly, the stimulation was always started at the right ventricular apex with burst pacing $(500-280 \mathrm{~ms})$ followed by a sequential insertion of single and double extrastimuli

Table 1 Selection of patients for participation in the study

\begin{tabular}{lrl}
\hline Selection process & Patients $(\boldsymbol{n})$ \\
\hline Patients admitted with acute Q wave MI & 164 & \\
Deaths in hospital & 17 & \\
Patients screened for study & 147 & \\
Exclusions from study: & 72 & $(49 \%)$ \\
Moderate to severe angina & 12 & \\
Killip class III or IV CHF & 16 & \\
Sustained VT & 3 & \\
End stage disease & 11 & \\
Patient refusal & 30 &. \\
Patients participating in study & $75 \quad(51 \%)$ \\
\hline
\end{tabular}

MI, myocardial infarction; CHF, congestive heart failure; VT, ventricular tachycardia. at paced cycle lengths of 500 and $400 \mathrm{~ms}$. If sustained ventricular tachycardia or fibrillation was not induced, the same steps were repeated at the right ventricular outflow tract and then at the left ventricular apex. Triple extrastimuli were used only after double extrastimuli at all three sites had failed to induce sustained arrhythmias.

Sustained ventricular tachycardia was defined as that which lasted 30 seconds or longer or caused haemodynamic compromise and had to be terminated in less than 30 seconds by direct current counter shock or overdrive pacing. Non-sustained ventricular tachycardia was defined as that which lasted from six beats to less than 30 seconds and did not cause haemodynamic compromise. Ventricular fibrillation was defined as a disorganised rapid ventricular activity requiring immediate defibrillation because of haemodynamic compromise.

Cardiac catheterisation with angiography was performed 15-30 minutes after completion of programmed ventricular stimulation. The criterion for a significant coronary artery stenosis was a reduction of $\geqslant 70 \%$ in the internal diameter of the vessel. Left ventricular angiograms were performed in the $30^{\circ}$ right anterior oblique ( 75 patients) and in the $55^{\circ}$ left anterior oblique projections (73 patients). A quantitative score of the left ventricular wall motion abnormality was determined in both the right anterior oblique and left anterior oblique projections by a previously described method. ${ }^{12}$ Each projection was divided into five segments and the contraction of each segment was scored as follows: normal $=1$, moderate hypokinesia $=2$, severe hypokinesia $=3$, akinesia $=4$, and dyskinesia $=5$. A quantitative score was obtained by adding the assigned value to each segment of the ventricle. With this system, a score of 10 equals normal wall motion function, 1015 indicates a slight abnormality, 15-25 a moderate abnormality, and $>25$ a severe wall motion abnormality of the left ventricle.

FOLLOW UP AND STATISTICAL ANALYSIS

Fifty two of the 75 patients were followed in the cardiology clinics of the Los Angeles CountyUniversity of Southern California Medical Center at regular intervals of 4-16 weeks. In others the follow up data were obtained by telephone either from the patient or from the patient's private physician. The study end points were death or the development of documented spontaneous sustained ventricular tachycardia or fibrillation. Sudden death was defined as death within an hour of the onset of terminal symptoms or that which occurred unexpectedly without any premonitory symptoms.

Values were expressed as mean (SD). Statistical analysis was performed by a $\chi^{2}$ test or $t$ test as 
appropriate. The actuarial probability curves were constructed according to the method of Kaplan and Meier. ${ }^{13}$ First, the univariate predictors of arrhythmic events were determined by a $\chi^{2}$ test. Then we used stepwise Cox regression analysis to determine variables of independent prognostic significance. The variables in this analysis included the following: presence or absence of congestive heart failure; site of acute myocardial infarction; left ventricular ejection fraction; left ventricular wall motion abnormality score; and inducibility of sustained ventricular tachycardia, ventricular fibrillation, or both.

\section{Results}

\section{RESPONSE TO PROGRAMMED VENTRICULAR} STIMULATION

Programmed ventricular stimulation induced sustained ventricular tachycardia in 19 patients and ventricular fibrillation in 14 patients. These 33 patients were assigned to group 1 . These arrhythmias were induced by right ventricular stimulation in 24 patients and left ventricular stimulation in nine patients. The cycle length of ventricular tachycardia ranged from 200 to $290 \mathrm{~ms}$ (mean $232(16) \mathrm{ms}$ ); the QRS complexes were of right bundle branch block configuration in 11 patients and of left bundle branch block configuration in eight patients. Sustained ventricular tachycardia was induced by two extrastimuli in 11 patients, three extrastimuli in seven patients, and burst pacing in one patient. Induction of ventricular fibrillation required two extrastimuli in six patients and three extrastimuli in eight patients.

Programmed ventricular stimulation did not induce sustained ventricular arrhythmias in 42 patients (group 2). In six of them non-sustained ventricular tachycardia was induced (uniform in four patients and multiform in two). Induction required two extrastimuli in two patients and triple extrastimuli in four patients.

Table 2 summarises the clinical, haemodynamic, and angiographic characteristics of patients in each group. Group 1 patients had an increased frequency of mild congestive heart failure, and a higher value for peak serum creatine kinase, pulmonary artery wedge pressure, and left ventricular wall motion abnormality score. However, the two groups did not significantly differ for 24 hour ambulatory electrocardiographic monitoring or treadmill testing, location of the infarct, left ventricular angiographic ejection fraction, and the frequency of left ventricular aneurysms.

FOLLOW UP OBSERVATIONS AND TREATMENT The follow up ranged from four to 36 (18(7)) months and was complete in all but five patients (two in group
Table 2 Profile of patients with inducible sustained ventricular tachycardia or fibrillation and without

\begin{tabular}{|c|c|c|c|}
\hline \multirow[b]{2}{*}{ Variable } & \multicolumn{2}{|c|}{ Sustained VT/VF } & \multirow[b]{2}{*}{ p value } \\
\hline & $\begin{array}{l}\text { Inducible } \\
(n=33) \\
\text { (group 1) }\end{array}$ & $\begin{array}{l}\text { Non-inducible } \\
(n=42) \\
(\text { group 2) }\end{array}$ & \\
\hline $\begin{array}{l}\text { Peak creatine } \\
\text { kinase (IU/1) } \\
\text { Mild CHF (Killip }\end{array}$ & 2347 (1739) & $1592(1426)$ & $<0.005$ \\
\hline $\begin{array}{l}\text { class II) } \\
\text { Anterior MI } \\
\text { PVCs/h`} \\
\text { PAW (mm Hg) } \\
\text { LV angiographic }\end{array}$ & $\begin{array}{r}10(33 \%) \\
15(45 \%) \\
326(1267) \\
16 \cdot 4(7 \cdot 6)\end{array}$ & $\begin{array}{c}4(10 \%) \\
21(50 \%) \\
282(631) \\
13 \cdot 3(5 \cdot 8)\end{array}$ & $\begin{array}{l}<0.01 \\
\text { NS } \\
\text { NS } \\
<0.05\end{array}$ \\
\hline $\begin{array}{l}\text { WMA score } \\
\text { LV ejection fraction } \\
\text { LV aneurysm } \\
\text { No of diseased }\end{array}$ & $\begin{array}{l}22(6) \\
0.47(0 \cdot 15) \\
12(36 \%)\end{array}$ & $\begin{array}{l}19(6) \\
0.48(0 \cdot 11) \\
11(26 \%)\end{array}$ & $\begin{array}{l}<0.05 \\
\text { NS } \\
\text { NS }\end{array}$ \\
\hline coronary arteries & $2 \cdot 2(0.4)$ & $2.1(0.3)$ & NS \\
\hline
\end{tabular}

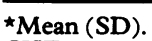

CHF, congestive heart failure; MI, myocardial infarction; PVCs, premature ventricular contractions; LV, left ventricular; PAW, pulmonary artery wedge pressure; WMA, wall motion abnormality.

1 and three in group 2) who moved out of the country 4-8 months after the index infarction. $\beta$ Adrenergic blockers were given to only seven patients (four in group 1 and three in group 2) for control of angina, hypertension, or both. Prophylactic antiarrhythmic treatment was not routinely administered except for the first 10 patients in group 1 who received procainamide or quinidine for the first $3-6$ months. Six patients in group 1 and five in group 2 underwent coronary artery bypass surgery, percutaneous transluminal coronary angioplasty, or both for left main coronary disease or for symptoms of intractable angina.

At the end of an average 18 months follow up, arrhythmic events had occurred in seven patients: these were sudden death in four patients and nonfatal spontaneous sustained ventricular tachycardia in three patients. None of the seven patients was on antiarrhythmic treatment at the time of the arrhythmic events, although two patients had received this treatment in the preceding year. One group 1 patient died of progressive heart failure 11 months after the infarction. Thus the overall total cardiac mortality was $6.7 \%$ (five of 75 patients) and the mortality caused by sudden death was $5 \cdot 3 \%$. In addition, nonfatal recurrent myocardial infarction developed in three group 1 patients and in one group 2 patient.

\section{INDUCIBILITY IN RELATION TO ARRHYTHMIC} EVENTS

There were arrhythmic events in five $(15 \%)$ of 33 patients in group 1 compared with two $(5 \%)$ of 44 patients in group $2(\mathrm{p}<0.4)$. The two year probability of remaining free of arrhythmic events was 0.83 for patients in group 1 and 0.95 in group 2 
(p $<0.4$ ) (fig 1a). Among group 1 patients, three patients died suddenly 15,16 , and 26 months after the index infarct and two patients had spontaneous sustained ventricular tachycardia at two weeks and 26 months after the infarct. Three extrastimuli were required to induce sustained arrhythmias in two of these five patients. Arrhythmic events did not develop in any of the nine patients in whom induction of sustained arrhythmias had required left ventricular stimulation. One patient in group 2 died suddenly three months after the index infarct and one had spontaneous sustained ventricular tachycardia two months after the infarct. The maximum induced response in both patients was less than three repetitive ventricular beats.

The risk of arrhythmic events was highest in a subset of 19 group 1 patients in whom sustained ventricular tachycardia was induced. Arrhythmic events occurred in four (three sudden deaths and one sustained ventricular tachycardia) of these 19 patients compared with three of the remaining 56 patients in whom arrhythmia was not inducible or in whom only ventricular fibrillation was induced. The
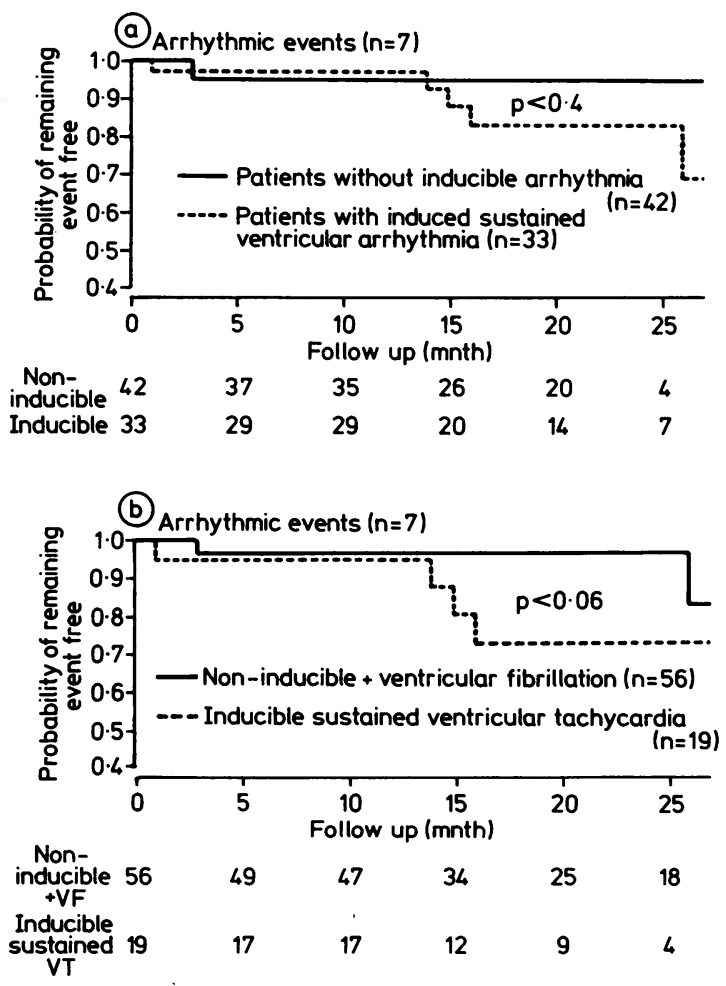

Fig 1 Prediction of sudden death and non-fatal spontaneous sustained ventricular tachycardia by programmed ventricular stimulation.
Table 3 Clinical, angiographic, and electrophysiological characteristics of patients with inducible sustained ventricular tachycardia who had or did not have arrhythmic events

\begin{tabular}{|c|c|c|c|}
\hline Variable & $\begin{array}{l}\text { Patients } \\
\text { with } \\
\text { arrhythmic } \\
\text { events } \\
(n=4)\end{array}$ & $\begin{array}{l}\text { Patients } \\
\text { without } \\
\text { arrhythmic } \\
\text { events } \\
(n=15)\end{array}$ & $\begin{array}{l}p \\
\text { value }\end{array}$ \\
\hline \multirow{5}{*}{ 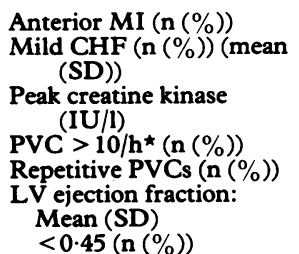 } & $2(50)$ & $5(33)$ & NS \\
\hline & $3(75)$ & $7(47)$ & NS \\
\hline & $3137(1245)$ & 2019 (1403) & NS \\
\hline & $\begin{array}{l}2(50) \\
3(75)\end{array}$ & $\begin{array}{l}2(13) \\
3(20)\end{array}$ & $\begin{array}{l}\text { NS } \\
<0.07\end{array}$ \\
\hline & $\begin{array}{l}0.38(0.9) \\
3(75)\end{array}$ & $\begin{array}{l}0 \cdot 42(0 \cdot 17) \\
8(53)\end{array}$ & $\begin{array}{l}\text { NS } \\
\text { NS }\end{array}$ \\
\hline $\begin{array}{l}\text { LVEDP (mm Hg) (mean } \\
\text { (SD)) }\end{array}$ & $20(8)$ & $23(8)$ & NS \\
\hline $\begin{array}{l}\text { LV WMA score (mean } \\
\text { (SD)) }\end{array}$ & $25(2)$ & $25(5)$ & NS \\
\hline $\begin{array}{l}\text { No coronary arteries } \\
\text { diseased (mean (SD)) } \\
\text { LV aneurysm (n (\%)) } \\
\text { VT cycle length: }\end{array}$ & $\begin{array}{l}2 \cdot 7(0 \cdot 5) \\
2(50)\end{array}$ & $\begin{array}{l}1 \cdot 9(0 \cdot 8) \\
6(40)\end{array}$ & $\begin{array}{l}0.09 \\
\text { NS }\end{array}$ \\
\hline $\begin{array}{l}\text { Mean (SD) (ms) } \\
>250 \mathrm{~ms}(\mathrm{n}(\%))\end{array}$ & $\begin{array}{r}252(24) \\
3(75)\end{array}$ & $\begin{array}{r}225(21) \\
3(20)\end{array}$ & $\begin{array}{r}<0.05 \\
0.07\end{array}$ \\
\hline $\begin{array}{l}\text { VT induction: } \\
\text { No of extrastimuli } \\
(\text { mean }(\text { SD }))\end{array}$ & $2 \cdot 25(0.5)$ & $2.73(0.7)$ & NS \\
\hline $\begin{array}{l}\text { By } \leqslant 2 \text { extrastimuli } \\
(\mathrm{n}(\%))\end{array}$ & $3(75)$ & $6(40)$ & NS \\
\hline
\end{tabular}

*Mean hourly frequency during 24 hour ambulatory electrocardiographic recording. EDP, end diastolic pressure. See footnote to table 2 for other abbreviations.

two year probability of remaining free of arrhythmic eventş was 0.73 and 0.95 respectively $(p<0.06)$ (fig 1b). We then compared the tachycardia characteristics and other clinical and angiographic features of these four patients who had arrhythmic events with those in the remaining 15 patients with inducible sustained ventricular tachycardia but no arrhythmic events (table 3 ). The mean cycle length of the tachycardia was significantly higher in patients with arrhythmic events than in those without $(252$ (24) $\mathrm{ms}$ $v 225(21) \mathrm{ms}, \mathrm{p}<0.05)$. Patients with arrhythmic events were more likely to have ventricular tachycardia induced by two extrastimuli, an increased number of diseased coronary arteries, and more repetitive ventricular extrasystoles and mild congestive heart failure.

For predicting subsequent arrhythmic events the inducibility of sustained ventricular arrhythmias had a sensitivity of $71 \%$, a specificity of $60 \%$, and a positive predictive accuracy of $15 \%$. When sustained ventricular tachycardia (and not ventricular fibrillation) was considered to be the only criterion for a positive response, the corresponding values were $57 \%, 78 \%$, and $21 \%$. 


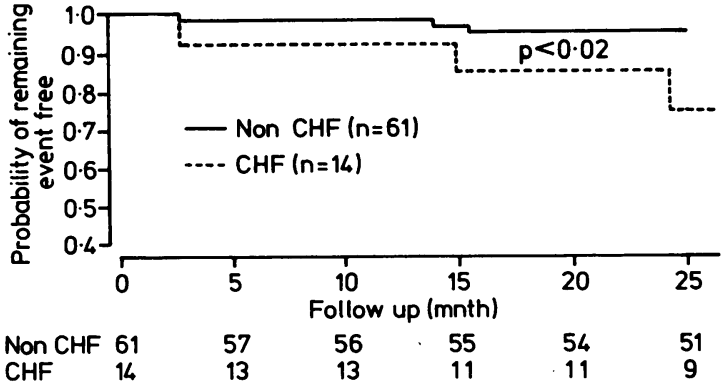

Fig 2 Prediction of sudden death and non-fatal spontaneous sustained ventricular tachycardia by the presence or absence of mild congestive heart failure (CHF).

\section{ARRHYTHMIC EVENTS IN RELATION TO OTHER ARIABLES}

A stepwise Cox regression analysis showed that mild congestive heart failure was the only significant predictor $(p<0.01)$ of the subsequent arrhythmic events. There were arrhythmic events in four $(29 \%)$ of the 14 patients with mild congestive heart failure and three $(5 \%)$ of the remaining 61 patients. The two year probability of remaining free of arrhythmic events was 0.76 in patients with and 0.94 in those without heart failure $(p<0.02)$ (fig 2 ). In the prediction of future arrhythmic events the presence of mild congestive heart failure had a sensitivity, specificity, and positive predictive accuracy of $57 \%$, $85 \%$, and $28 \%$ respectively.

Among group 1 patients there was no significant difference in the occurrence of arrhythmic events in patients who had received previous antiarrhythmic treatment and those who had not $(20 \% v 13 \%$, $\mathrm{p}=\mathrm{NS}$ ). None of the 11 patients (six group 1 and five group 2) who had procedures for coronary reperfusion developed arrhythmic events during follow up.

\section{Discussion}

This prospective study was designed to determine the prognostic significance of programmed ventricular stimulation in clinically stable survivors of an acute myocardial infarction. The overall total cardiac mortality was low (6.7\%) after an average follow up of 18 months, and undoubtedly reflects the exclusion of high risk patients. In this low risk group, programmed ventricular stimulation performed two weeks after infarction induced sustained ventricular tachycardia or fibrillation in $44 \%$ of the patients. The incidence of inducibility was higher than that (20 to $33 \%$ ) reported elsewhere $e^{5-1012}$ and was probably related to the vigor of the stimulation protocol in our study. We used a maximum of three extrastimuli delivered at both right and left ventricles. In con- $c$. trast, in most of the earlier studies a maximum of two $\vec{F}$ extrastimuli was used and left ventricular stimulation $\stackrel{5}{?}$ was not performed. The inducibility of sustained ventricular arrhythmias correlated with the indices $\frac{\bar{F}}{\bar{N}}$ of a larger infarct size, such as peak creatine kinase $\frac{\text { ? }}{\overrightarrow{0}}$ activities, the presence of congestive heart failure, $\AA$ and the left ventricular angiographic wall motion $ळ$ abnormalities. These findings accord with those $\vec{\circ}$ previously reported in survivors of acute myocardial infarction. ${ }^{-12}$

The inducibility of sustained ventricular arrhyth- $\frac{\rho}{P}$ mias did not predict future arrhythmic events. These events occurred in $15 \%$ of patients with inducible 9 sustained arrhythimias and $5 \%$ of those without ir $(p=0.4)$. The prognosis was best related to the presence or absence of mild congestive heart failure $(29 \% v 5 \%, p<0.02)$. These findings accord with $\frac{}{5}$ those of three previous studies that evaluated the prognostic significance of programmed ventricular stimulation in clinically stable survivors of acute myocardial infarction. ${ }^{7-9}$ In these studies, however, $\mathscr{\odot}$ the stimulation protocol included a maximum of two extrastimuli at twice diastolic threshold; conceivably, 응 the lack of programmed ventricular stimulation may have lacked predictive value because the stimulation $\bar{\partial}$ protocol was inadequate. Indeed, in our study, two of $\stackrel{2}{\circ}$ the five group 1 patients in whom arrhythmic events $\stackrel{\mathscr{Q}}{\triangle}$ developed had required triple extrastimuli for induc- $\overrightarrow{\vec{F}}$ tion of sustained arrhythmia, but triple extrastimuli $\stackrel{\circ}{3}$ also resulted in a much higher incidence $(44 \%)$ of $\bar{P}$ inducibility. Arrhythmic events did not develop in any of the nine group 1 patients who had required left ventricular stimulation for induction of sustained $\stackrel{\mathbb{D}}{\mathscr{D}}$ ventricular tachycardia or ventricular fibrillation.

Our findings differ from those of Denniss et al who performed programmed ventricular stimulation in 403 clinically stable patients with acute myocardial $\stackrel{3}{3}$ infarction. ${ }^{11}$ They induced ventricular tachycardia (of longer than 10 seconds) or ventricular fibrillation in $34 \%$ of the patients and found that the two year $\frac{D}{0}$ probability of remaining free of arrhythmic events was significantly lower in patients with inducible $\tilde{\sigma}$ ventricular tachycardia or ventricular fibrillation $N$ than in those without $(0.84 v 0.95, \mathrm{p}<0.01)$. In the two studies, however, patient characteristics and ${ }_{\sigma}^{\omega}$ protocols for programmed ventricular stimulation differed. In our study, $51 \%$ of the surviving patients $\stackrel{\circ}{=}$ after infarction entered the study, whereas the study $\mathscr{D}$ by Denniss et al included $81 \%$ of all survivors of acute myocardial infarction. Their study undoubtedly included more of the high risk patients. Cardiac death or non-fatal sustained ventricular $\underset{\mathbb{D}}{\mathbb{D}}$ arrhythmias developed in $9 \%$ of their patients during $\cong$ a follow up of 12 months compared with $7.3 \%$ of our 
patients after an average follow up of 18 months. In addition, Denniss et al used a non-standard protocol for programmed ventricular stimulation that included delivery of two extrastimuli at a high current strength of $20 \mathrm{~mA}$. The available data on the specificity and sensitivity of this protocol are limited and controversial. ${ }^{14} 15$ In contrast, the value of the protocol used in our study is well established for several clinical conditions, including patients with recurrent sustained ventricular tachycardia or cardiac arrest. ${ }^{16-18}$

The configuration of the induced arrhythmia seemed to be of prognostic significance. Four of the 19 patients with inducible uniform sustained ventricular tachycardia had an arrhythmic event during follow up compared with three of the remaining 56 patients $(21 \% v 5 \%, p<0.06)$. In contrast, induction of ventricular fibrillation seemed to be a nonspecific response as only one of the 14 patients with this response had an arrhythmic event during follow up. Similar observations have recently been reported by Denniss et al who did not find an increased risk of arrhythmic events in patients with inducible ventricular fibrillation compared with those with no inducible arrhythmia $(8 \% v 7 \%, \mathrm{p}=\mathrm{NS}) .^{11}$ In contrast, inducible ventricular tachycardia identified a subset $(20 \%$ of all patients) that had a $27 \%$ probability of developing arrhythmic events within two years of infarction. In both studies, the patients with inducible ventricular tachycardia who developed arrhythmic events tended to have a longer cycle length for the induced ventricular tachycardia and a lower left ventricular ejection fraction. These findings were not unexpected and accord with the experimental observations in a canine model of myocardial infarction. ${ }^{19}$ During serial programmed ventricular stimulation in 20 anaesthetised dogs, Garan et al showed a high week to week reproducibility $(>90 \%)$ of inducible sustained uniform ventricular tachycardia, whereas induction of ventricular fibrillation was found to be a nonspecific response. ${ }^{19}$ These findings also accord with observations in human beings where day to day reproducibility has been shown to be high for sustained ventricular tachycardia and low for ventricular fibrillation induced in the post-infarction period. ${ }^{20}$

\section{CLINICAL IMPLICATIONS}

To be useful for identifying patients at risk of arrhythmic events, a test should have a high sensitivity and positive predictive accuracy and should also provide prognostic information independent of a routine clinical evaluation. In the present study, inducible sustained ventricular tachycardia had a low sensitivity $(57 \%)$ and positive predictive accuracy
$(21 \%)$ for future arrhythmic events. Instead, outcome was best predicted by the presence or absence of mild congestive heart failure. Had we excluded the 14 patients with mild congestive heart failure from our study, programmed ventricular stimulation in the remaining 61 patients would have identified only one patient in whom an arrhythmic event developed. In previous prospective studies of programmed ventricular stimulation, the positive predictive accuracy of programmed ventricular stimulation was $18 \%$ and $32 \%,{ }^{11}$ and no study so far has shown the inducible arrhythmia to be an independent predictor of arrhythmic events. Moreover, programmed ventricular stimulation is an invasive and expensive test associated with a small but definite morbidity which can be performed at only a few laboratories with a highly trained staff. For these reasons, we believe that the routine use of this procedure is not recommended in clinically stable patients surviving an acute myocardial infarction. However, programmed ventricular stimulation may be of more value in patients whose post-infarction course is complicated by heart failure, angina pectoris, or ventricular tachycardia. A prospective study in such a high risk subset is already in progress at our medical centre. Among the first 53 patients studied, major arrhythmic events occurred in five of 10 patients with inducible sustained ventricular tachycardia and two of $43(4.8 \%)$ patients in whom ventricular arrhythmias were non-inducible or who had induction of ventricular fibrillation $(p<0.01){ }^{21}$ These preliminary findings, though encouraging, need to be confirmed in a larger cohort of patients.

\section{CRITIQUE OF THE STUDY}

In this study, 10 of the 33 patients with inducible sustained arrhythmias received empirical antiarrhythmic treatment for the first 3-6 months, and this might have influenced outcome. However, no previous study has shown a beneficial effect of prophylactic antiarrhythmic treatment on survival in such patients. Moreover, antiarrhythmic treatment was not based on the results of electropharmacological testing, and two of these 10 patients had an arrhythmic event during follow up. $\beta$ Adrenergic blocking agents were administered to only seven patients for control of angina or systemic hypertension. The outcome of the study population might also have been altered by the coronary artery bypass surgery, percutaneous transluminal coronary angioplasty, or both, which were performed in 11 patients $(15 \%)$. Although none of these 11 patients had an arrhythmic event during follow up, the number of patients undergoing coronary reperfusion was too small to draw a meaningful conclusion. Also, there was no significant difference in the proportion of 
patients undergoing myocardial revascularisation between the two groups.

Supported by a grant from the GCRC Program of the Division of Research Resources, National Institute of Health.

\section{References}

1 The Multicenter Post-Infarction Research Group. Risk stratification and survival after myocardial infarction. N Engl J Med 1983;309:331-6.

2 Mukharji J, Rude RE, Poole KW, and the MILIS Study Group. Risk factors for sudden death after acute myocardial infarction: two year follow-up. $\mathrm{Am} \mathrm{J}$ Cardiol 1984;54:31-6.

3 Roubin GS, Harris PJ, Bernstein L, Kelly DT. Coronary anatomy and prognosis after myocardial infarction in patients 60 years of age and younger. Circulation 1983;67:743-9.

4 Hamer A, Vohra J, Hunt D, Sloman G. Prediction of sudden death by electrophysiologic studies in high risk patients surviving acute myocardial infarction. Am J Cardiol 1982;50:223-9.

5 Waspe LE, Seinfeld D, Ferrick A, Kim SG, Matos JA, Fisher JD. Prediction of sudden death and spontaneous ventricular tachycardia in survivors of complicated myocardial infarction: value of the response to programmed stimulation using a maximum of three ventricular extrastimuli. J Am Coll Cardiol 1985;5: 1292-301.

6 Roy D, Marchand E, Theroux P, Waters D, Pelletier $G$, Bourassa M. Programmed ventricular stimulation in survivors of an acute myocardial infarction. Circulation 1985;72:487-94.

7 Breithardt G, Borggrefe $M$, Haerten K. Role of programmed ventricular stimulation and noninvasive recording of ventricular late potentials for the identification of patients at risk of ventricular tachyarrhythmias after acute myocardial infarction. In: Zipes DP, Jalife J, eds. Cardiac electrophysiology and arrhythmias. New York: Grune and Stratton, 1985: 553-61.

8 Marchlinski FE, Buxton AE, Waxman HL, Josephson ME. Identifying patients at risk of sudden death after myocardial infarction: value of the response to programmed stimulation, degree of ventricular ectopic activity and severity of left ventricular dysfunction. Am J Cardiol 1983;52:1190-6.

9 Santarelli P, Bellocci F, Loperfido F, et al. Ventricular arrhythmia induced by programmed ventricular stimulation after acute myocardial infarction. $A m J$ Cardiol 1985;55:391-4.

10 Bhandari AK, Rose JS, Kotlewski A, Rahimtoola SH,
Wu D. Frequency and significance of induced sus- $\frac{\stackrel{D}{\beth}}{\beth}$ tained ventricular tachycardia or fibrillation two $c$. weeks after acute myocardial infarction. $\mathrm{Am} \mathrm{J} \mathrm{Cardiol} \rightrightarrows$ 1985;56:737-42.

11 Denniss AR, Richards DA, Cody DV, et al. Prognostic significance of ventricular tachycardia and fibrillation $\bar{\sigma}$ induced at programmed stimulation and delayed $\overline{\bar{\omega}}$. potentials detected on the signal-averaged electrocar- $\mathbb{\Phi}$ diograms of survivors of acute myocardial infarction. Circulation 1986;74:731-45.

12 CASS Investigators. National Heart, Lung and Blood $\vec{\circ}$ Institute Coronary Artery Surgery Study: Appendix C-Left ventriculography. Circulation 1981;63 $\vec{\omega}$ (suppl I):164-5.

13 Kaplan EL, Meier P. Nonparametric estimation from incomplete observations. J Am Stat Assoc 1985;53:0ं 457-81.

14 Richards DA, Cody DV, Denniss AR, Russell PA, i Young AA, Uther JB. A new protocol of programmed $\vec{O}$ stimulation for assessment of predisposition to spon-o taneous ventricular arrhythmias. Eur Heart J 1983; 4:376-82.

15 Brugada P, Wellens HJJ. Comparison in the sameô patient of two programmed ventricular stimulation protocols to induce ventricular tachycardia. $\mathrm{Am} J \overrightarrow{\mathrm{O}}$ Cardiol 1985;55:380-3.

16 Brugada P, Green M, Abdollah H, Wellens HJJ. Significance of ventricular arrhythmias initiated by programmed ventricular stimulation: the importance of the type of ventricular arrhythmias induced and the number of premature stimuli required. Circulation 1984;69:87-92.

17 Kudenchuck PJ, Kron J, Walance CG, et al. $\vec{F}$ Reproducibility of arrhythmia induction with윽 intracardiac electrophysiologic testing: patients with clinical sustained ventricular tachyarrhythmias. $J A m$ Coll Cardiol 1986;7:819-28.

18 Mason J, Winkle R. Electrode-catheter arrhythmia induction in the selection and assessment of antiarrhythmic drug therapy in recurrent ventricular ${ }_{\sigma}^{-}$ tachycardia. Circulation 1978;58:971-85.

19 Garan H, Ruskin J, McGovern B, Grant B. Serial analysis of electrically induced ventricular arrhyth-윽 mias in a canine model of myocardial infarction. $J \mathrm{Am}_{\mathrm{O}}$ Coll Cardiol 1985;5:1095-106.

20 Hong R, Bhandari A, Kulick D, Rubin JN, McIntosh D N, Rahimtoola SH. Day-to-day reproducibility of response to programmed ventricular stimulation in patients with recent acute myocardial infarction. [Abstract]. J Am Coll Cardiol 1988;11:5A.

21 Bhandari A, Hong R, Kotlewski A, Rubin JN, N McIntosh N, Rahimtoola SH. Prognostic significance of programmed stimulation in high risk patients surviving acute myocardial infarction [Abstract] J Am Coll Cardiol 1988;11:6A. 